\title{
Study of the molecular variation in pre-eclampsia placenta based on micro-Raman spectroscopy
}

\author{
Si-Jin Chen $\cdot$ Yuan Zhang $\cdot$ Xiang-Ping Ye $\cdot$ Kun Hu \\ Mei-Fang Zhu $\cdot$ Yan-Yue Huang $\cdot$ Mei Zhong $\cdot$ \\ Zheng-Fei Zhuang
}

Received: 25 November 2013 / Accepted: 5 May 2014 / Published online: 28 May 2014

(C) The Author(s) 2014. This article is published with open access at Springerlink.com

\begin{abstract}
Purpose Study of the molecular variation in pre-eclampsia placenta based on micro-Raman spectroscopy.

Methods Five pregnant women with pre-eclampsia from Nanfang hospital were selected as study group whose average age is 28.5 years and $38 \pm 2$ weeks gestation. The same period of healthy pregnant women, whose average age is 27.6 years and pregnant $39 \pm 1$ weeks, as control group $(n=5)$. The normal and pre-eclamptic placental tissues are detected by micro-Raman spectroscopy with the spectrum resolution of $1 \mathrm{~cm}^{-1}$.

Results We find that the protein structure of $\alpha$-helix, $\beta$-pleated sheet and $\beta$-turn is overlying in pre-eclamptic placenta, which lead to a disorder of protein structure. The Raman peaks assigned to tryptophan indole ring and phenylalanine in pre-eclamptic placental tissue are more higher than that in normal tissue.
\end{abstract}

\section{S.-J. Chen and Y. Zhang contributed equally to this study.}

S.-J. Chen · M.-F. Zhu · Z.-F. Zhuang ( $\square)$

MOE Key Laboratory of Laser Life Science and Laboratory of Photonic Chinese Medicine, College of Biophotonics, South China Normal University, Guangzhou 510631, China

e-mail: zhuangzf@scnu.edu.cn

\section{S.-J. Chen $\cdot$ Y. Zhang $\cdot$ M. Zhong $(\bowtie)$}

Department of Obstetrics and Gynecology, Nanfang Hospital, Southern Medical University, Guangzhou 510515, China e-mail: zhongmei@smu.edu.cn

\section{X.-P. Ye $\cdot$ K. Hu}

National Testing Center for Optical Radiation Safety of Photoelectric Products, Huizhou 516003, China

Y.-Y. Huang

Laboratory of Photonic Information Technology, South China

Normal University, Guangzhou 510631, China
Conclusions Results suggest that the ordered structures of the main chain in protein molecules are reduced significantly, and the amino acid of side chains is damaged obviously. And a principal component analysis is used to classify the Raman spectra between normal and pre-eclamptic placental tissues. This study presents that Raman spectroscopy has a great potential on the mechanism research and diagnosis of placental lesions.

Keywords Molecular variation · Pre-eclampsia placenta . Micro-Raman spectroscopy · Principal component analysis $\cdot$ Scores plots

\section{Introduction}

Pre-eclampsia (also known as pregnancy-induced hypertension, PIH) is a disorder that generally develops late in pregnancy and is characterized by a sudden onset of high blood pressure, edema and protein in the urine [1]. However, the immunity, impaired vascular endothelial cells and insulin resistance etiology in this disease are unclear [2]. Researchers are interested in the etiology and pathology of pre-eclampsia. Turner et al. [3] observed that the concentration of tyrosine, histidine, and phenylalanine in pre-eclampsia placenta was higher than normal tissue. And Raouf et al. [4] reported that there was a decreased intensity at the protein bands from the Fourier spectra of lyophilized serum samples. There were some conflicting views in the previous studies [3, 4]. Therefore, an in-depth research on the molecular and protein variation of pre-eclampsia is urgently needed for scientists.

Confocal micro-Raman spectroscopy, which depends on polarizability change of an oscillating molecule, has proven extremely versatile and has led to a vast array of 
applications across the disciplines of chemistry, physics, biology, biomedicine, engineering, and archeology. With an insensitivity to the presence of water, Raman spectroscopy is a particularly attractive technique for life sciences. In addition to these, compared with other optics technologies including second harmonic generation, Fourier transform infrared spectroscopy (FTIR), and multiphoton microscopy, Raman spectroscopy requires simple sample preparation and contains abundant information [5-8]. With the help of these superiorities, Raman spectroscopy has been employed to detect the damage of gestational diabetes on placenta [9]. However, there were few reports about placenta in pre-eclampsia. In this study, the micro-Raman spectra were employed to characterize the molecular variation in pre-eclamptic placenta tissues.

\section{Materials and methods}

\section{Sample collection and preparation}

Informed consent for the study was obtained from all the women concerned, and approval was given by the Ethics Committee of the Faculty of Medicine, Nanfang Hospital, Southern Medical University. In this study, five pregnant women with pre-eclampsia from Nanfang hospital were selected as study group whose average age is 28.5 years and $38 \pm 2$ weeks gestation. The same period of healthy pregnant women, whose average age 27.6 years and pregnant $39 \pm 1$ weeks, as control group $(n=5)$. All of the placentas were obtained immediately after cesarean deliveries. There were no other obstetric complications in the two groups of pregnant women.

\section{Experiment}

Tissue samples were placed on a silicon slice for measurement of Raman spectroscopy because there were no extra Raman peaks for the silicon slice in the fingerprint spectrum region from 700 to $1,800 \mathrm{~cm}^{-1}$. The Raman spectra were acquired using a Renishaw (New Mills, UK) inVia confocal micro-Raman spectroscopy system. The samples were excited by $785 \mathrm{~nm}$ laser and after attenuation through prisms and filter, the power of the laser exposed on the samples was $9 \mathrm{~mW}$. Spectra were obtained from tissues with a $20 \times$ optimized objective and the signal was integrated for five times over a spectral range of $700-1,800 \mathrm{~cm}^{-1}$. Peak frequencies are calibrated with the silicon at $520 \mathrm{~cm}^{-1}$. For each sample, at least 20 Raman spectra are obtained. All the data are collected under the same conditions. Tissue samples were placed on a silicon slice for Raman measurement. In order to compare the related spectra changes, the phenylalanine band $\left(1,004 \mathrm{~cm}^{-1}\right)$ was chosen to normalize the spectra. The final Raman spectra are baseline corrected by the software R 2.8.1, and smoothed, normalized, and averaged by ORIGIN PRO 8.5 (OriginLab Corporation, Northampton, MA, USA), together with the Raman spectroscopic software WIRE 3.2.

Data analysis

The spectra recorded from normal and pre-eclamptic placental tissue were analyzed statistically using principal component analysis (PCA) by Matlab [6, 10, 11]. The analysis is oriented toward modeling a variance-covariance structure of a data matrix from which the eigenvalues, corresponding to principal components, are extracted. Each principal component (PC) is a linear combination of the $\mathrm{n}$ independent wavenumber variables $x_{1}, \ldots, x_{\mathrm{n}}$. So, for example:

$\mathrm{PC} 1=a_{1} x_{1}+a_{2} x_{2}+\cdots+a_{n} x_{n}$.

The first PC accounts for the greatest variance, and so corresponds to the largest eigenvalue. The second $\mathrm{PC}$ is orthogonal to the first, with each successive PC being both orthogonal to all those preceding, and accounting for a decreasing proportion of the variance. In this paper, we choose the first three PC for analysis (Fig. 1).

\section{Results}

A typical Raman spectrum of normal placenta is shown in Fig. 2. We can find that for normal placenta, primary Raman peaks were observed at 758, 940, 1,005, 1,033, 1,343, $1,453,1,605,1,620$ and $1,663 \mathrm{~cm}^{-1}$. The Raman peak of $1,453 \mathrm{~cm}^{-1}$ belonged to $\mathrm{CH}_{3}\left(\mathrm{CH}_{2}\right)$ deformation vibration of proteins. While $1,663 \mathrm{~cm}^{-1}$ is assigned to amide I, the bands at 758 and $1,343 \mathrm{~cm}^{-1}$ should be assigned to tryptophan

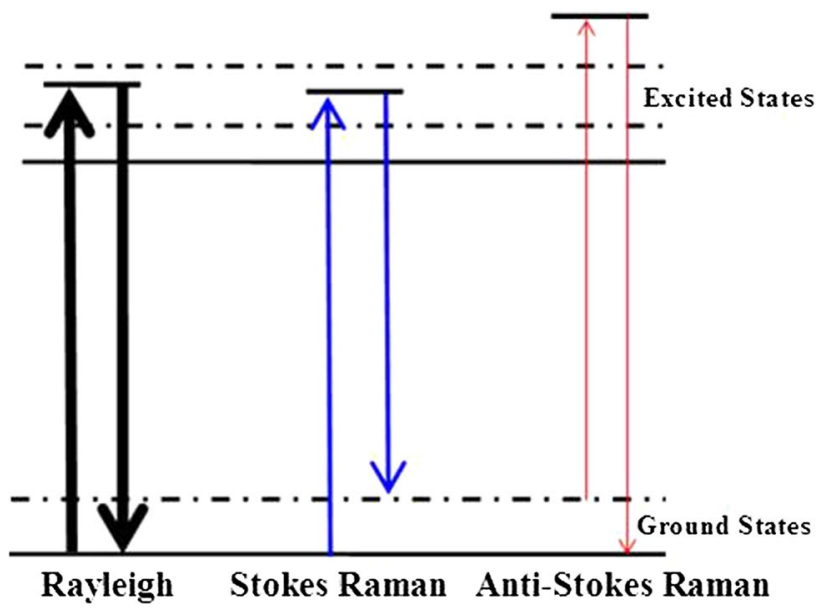

Fig. 1 Diagrammatic Raman scattering effect 


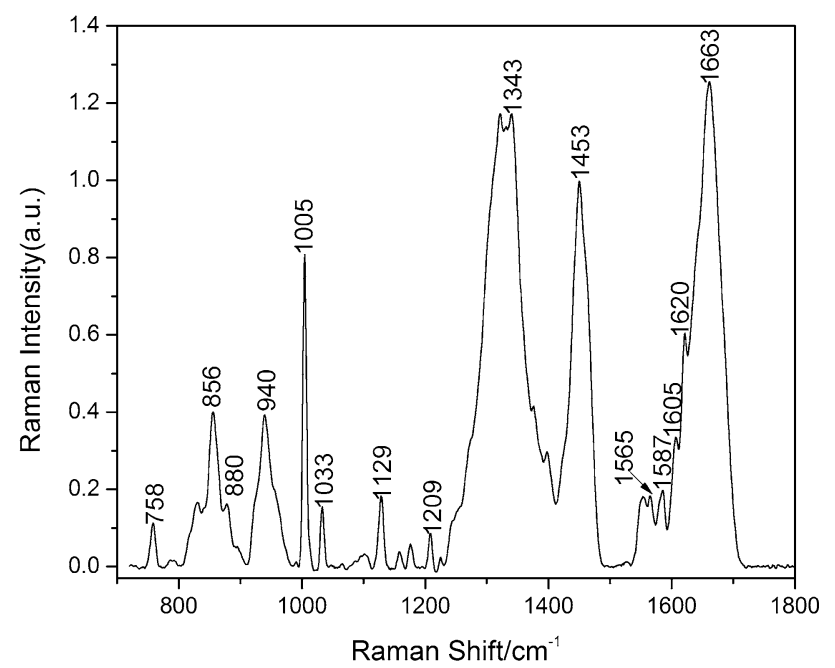

Fig. 2 The Raman spectrum of normal placenta

Table 1 Raman shift and the tentative assignment of normal placenta

\begin{tabular}{llll}
\hline $\begin{array}{l}\text { Frequency } \\
\left(\mathrm{cm}^{-1}\right)\end{array}$ & Assignment & $\begin{array}{l}\text { Frequency } \\
\left(\mathrm{cm}^{-1}\right)\end{array}$ & Assignment \\
\hline 758 & Trp & 856 & Tyr \\
880 & Trp & 940 & $\nu_{\mathrm{s}}\left(\mathrm{PO}_{3}{ }^{2-}\right)$ \\
1,005 & Phenylalanine & 1,033 & $\mathrm{Phe}$ \\
1,129 & $\nu(\mathrm{C}-\mathrm{N})$ & 1,159 & $\delta(\mathrm{C}-\mathrm{O})$ \\
1,175 & Tyr, Phe & 1,209 & Tyr \\
1,343 & Trp & 1,399 & His \\
1,453 & $\delta\left(\mathrm{CH}_{2}\right)$ & $1,549,1,565$ & Trp \\
1,585 & Trp & 1,605 & Phe \\
1,620 & Phe & 1,663 & Amide I \\
& & & protein \\
\hline
\end{tabular}

$v$ stretch, $v_{\mathrm{s}}$ symmetry stretch, $\operatorname{Tr} p$ tryptophan, Tyr tyrosine, Phe Phenylalanine, $\delta$ deformation, His histidine

indole ring, and Raman peaks at 1,005, 1,033, 1,605 and $1,620 \mathrm{~cm}^{-1}$ belong to phenylalanine. And the assignments of all the main Raman peaks are shown in Table 1.

This study mainly discusses the differences in the region of $1,500-1,700 \mathrm{~cm}^{-1}$ in Fig. 3. Figure 3a depicts Raman spectrum of normal placenta while Fig. $3 \mathrm{~b}$ shows the Raman spectrum of pre-eclamptic placenta. There are many differences between the Raman spectra of preeclamptic and normal placenta tissue. By contrast in Fig. 3, we can see that the intensity of peaks at 1,005, 1,605 and $1,620 \mathrm{~cm}^{-1}$ increased which belong to phenylalanine, while 785 and $1,585 \mathrm{~cm}^{-1}$ also enhanced which assigned to indole ring of tryptophan. The spectral line of amide I at $1,663 \mathrm{~cm}^{-1}$ from normal placenta presented $\alpha$-helix structure, but in the Raman spectrum of pre-eclamptic tissue, peaks at 1,662 and 1,640 $\mathrm{cm}^{-1}$ showed a overlying of $\alpha$-helix, $\beta$-pleated sheet and $\beta$-turn.

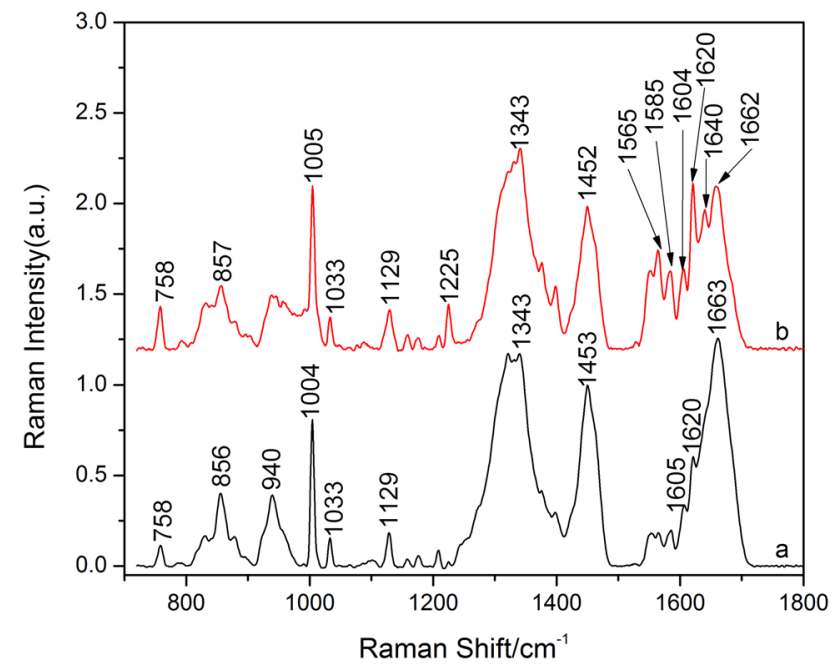

Fig. 3 The Raman spectra of normal and pre-eclamptic placenta. a Normal placenta. b Pre-eclamptic placenta

Finally, PCA was used to distinguish the Raman spectra of normal and pre-eclamptic placental tissues, which is shown in Fig. 4. In this section, 21 Raman spectra of pre-eclamptic placental tissue and 20 Raman spectra of normal placenta were used for PCA. We formed the variance-covariance matrix of the dataset, in which the eigenvalues or factors corresponding to PCs were extracted and the resulting scores were noted. The panel in Fig. 4 shows a plot of sample number vs. scores of PC1 vs. PC2, which represents the discrimination between spectra of normal and pre-eclamptic placental tissues. This plot clearly shows that most of the normal placentas lie in the first and fourth quadrants while most of the pre-eclamptic placental tissues lie in the second and third quadrants except two points. It could give us a useful help on qualitative distinction the normal and pre-eclamptic placental tissues.

\section{Discussion}

By comparing the Raman spectra of normal and preeclamptic placental tissues, it was clear that the characteristic spectral line of amide I at $1,663 \mathrm{~cm}^{-1}$ from normal placenta assigned to $\alpha$-helix structure, but peaks at 1,662 and $1,640 \mathrm{~cm}^{-1}$ in pre-eclamptic placental tissue showed a superposition composed by $\alpha$-helix, $\beta$-pleated sheet and $\beta$-turn which led to a disorder of protein structure [12]. Meanwhile, the Raman spectra also showed variations of amino acid residue on the protein side chain. In preeclamptic placenta, we observed significant enhanced spectral lines of tryptophan indole ring and phenylalanine and some special lines at 1,399 and $1,640 \mathrm{~cm}^{-1}$ which cannot be found in normal placental tissue, which shows an addition 


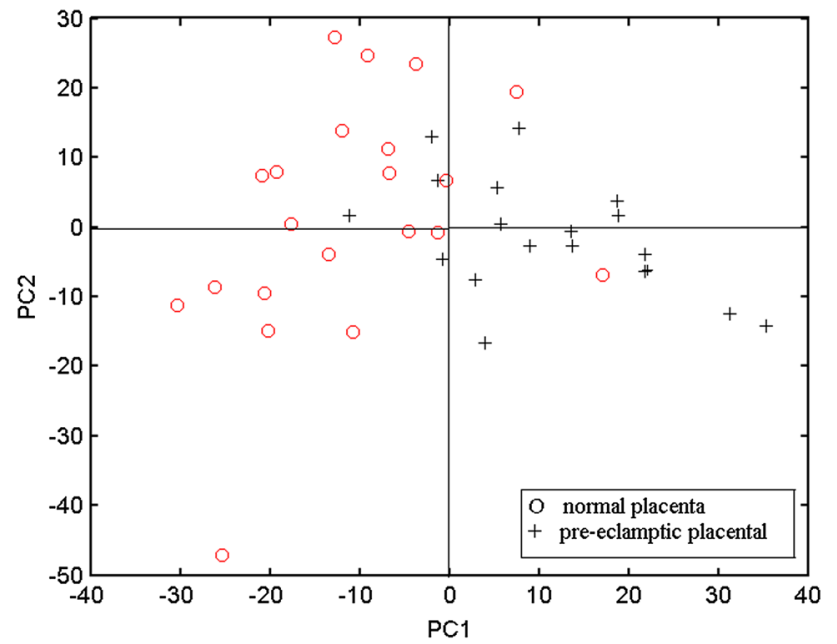

Fig. 4 Score plots of PCA

of the content of amino acid residue in pre-eclamptic placenta. The vasoactive substances synthesized and secreted by hypoxic-ischemic placenta (such as soluble Fms-like tyrosine kinase 1, cytokines, angiotensin II type 1 receptor activating antibody and thromboxane) entered into the maternal blood circulation and caused vascular endothelial cell dysfunction, which can further promote the release of vasoactive substances to decrease the activity of NO and increase reactive oxygen species and free radicals. Oxidation changed the main chain conformation and injured the side chain groups of protein which presented characteristic enhanced spectral lines of phenylalanine at 1,005, 1,605 and $1,620 \mathrm{~cm}^{-1}$ and those amines with activity side chain such as tryptophan indole ring at 758 and $1,585 \mathrm{~cm}^{-1}[13,14]$.

\section{Conclusion}

In this study, the results show that: (1) the protein structure of $\alpha$-helix, $\beta$-pleated sheet and $\beta$-turn is overlying in pre-eclamptic placenta, which lead to a disorder of protein structure. (2) The Raman peaks assigned to tryptophan indole ring and phenylalanine in pre-eclamptic placental tissue are more higher than that in normal tissue. It suggests that the ordered structures of the main chain in protein molecules are reduced significantly, and the amino acid of side chains is damaged obviously. (3) The PCA could give us a useful help on distinguishing the Raman spectra between normal and pre-eclamptic placental tissues. And the Raman spectroscopy presents a great potential on the mechanism research and diagnosis of placental lesions.

Acknowledgments This work was supported by the Specialized Research Fund for the Doctoral Program of Higher Education of China (No. 20134407120003), the PhD Startup Fund of
Natural Science Foundation of Guangdong Province, China (No. S2013040016223), the National Natural Science Foundation of China (No. 61275187, 11374107), and the Young Teachers Nurturing Fund of South China Normal University (2012KJ020) and the Horizontal Topic Research Project of SENSING.

Conflict of interest None.

Open Access This article is distributed under the terms of the Creative Commons Attribution License which permits any use, distribution, and reproduction in any medium, provided the original author(s) and the source are credited.

\section{References}

1. Siamak S, Arie F, Annemarieke R, Diederick EG, Mathias P, Marie-Louise B, Yvonne TS, Michiel LB (2007) High blood pressure in pregnancy and coronary calcification. Hypertension 49:813-817

2. Basar G, Parlatan U, Seninak S, Gunel T, Benian A, Kalelioglu I (2012) Investigation of preeclampsia using raman spectroscopy. J Raman Spectrosc 27(4):239-252

3. Turner E, Brewster JA, Simpson NAB, Walker JJ, Fisher J (2008) Aromatic amino acid biomarkers of preeclampsia-a nuclear magnetic resonance investigation. Hyperten- sion Pregnancy 27(3):225-235

4. Raouf GA, Al-Malki ARL, Mansouri N, Mahmoudi RM (2011) Preliminary study in diagnosis and early prediction of preeclampsia by using FTIR spectroscopy technique. Life Science J 8(2):453-464

5. Zhuang Z, Zhu M, Guo Z, Xiong K, Li N, Chen S (2012) Study of molecule variation in various stages of human nuclear cataracts by micro-Raman spectroscopy. Appl Phys Lett 101:173701

6. Zhuang Z, Li N, Guo Z, Zhu M, Xiong K, Chen S (2013) Study of molecule variations in renal tumor based on confocal microRaman spectroscopy. J Biomed Opt 18(3):031103

7. Zhuo S, Chen J, Wu G, Xie S, Zheng L, Jiang X, Zhu X (2012) Quantitatively linking collagen alteration and epithelial tumor progression by second harmonic generation microscopy. Appl Phys Lett 96(21):213704-213704-3

8. Zhuang Z, Liu H, Guo Z, Zhuo S, Yu B, Deng X (2010) Secondharmonic generation as a DNA malignancy indicator of prostate glandular epithelial cells. Chin Phys B 19(4):049501-049501-5

9. Yu G, Xu XX, Niu Y, Wang B, Song ZF, Zhang CP (2004) Studies on human breast cancer tissues with Raman microspectroscopy. Spectrosc Spect Anal 24(11):1359-1362

10. Wood BR, Caspers P, Puppels GJ, Pandiancherri S, McNaughton D (2007) Resonance Raman spectroscopy of red blood cells using near infrared excitation. Anal Bioanal Chem 387:1691-1703

11. Zachariah E, Bankapur A, Santhosh C, Valiathan M, Mathur D (2010) Probing oxidative stress in single erythrocytes with Raman Tweezers. J Photochem Photobiol B 100:113-116

12. Wang L, Fan JH, Guan ZF, Liu Y, Zeng J, He DL, Huang LQ, Wang XY, Gong HL (2012) Study on bladder cancer tissues with Raman spectroscopy. Spectrosc Spect Anal 32(1):123-126

13. Hertig A, Berkane N, Lefevre GK, Toumi HP, Marti J, Capeau S, Uzan E (2004) Rondeau maternal serum sflt1 concentration is an early and reliable predictive marker of preeclampsia. Clin Chem 50(9):1701-1703

14. Noris M, Perico N, Remuzzi G (2005) Mechanisms of disease: pre-eclampsia. Nat Clin Pract Nephr 1(2):98-114 